Research Article

\title{
The Comparison of Performance between Contrast Source Inversion and Its Related Algorithms and Several Improved Methods
}

\author{
Meng Wang $(\mathbb{D}$ and Guizhen Lu $\mathbb{D}$ \\ College of Information and Communication Engineering, Communication University of China, Beijing 100024, China \\ Correspondence should be addressed to Meng Wang; wangmeng1993@cuc.edu.cn
}

Received 21 May 2021; Revised 8 July 2021; Accepted 16 July 2021; Published 28 July 2021

Academic Editor: Stefano Selleri

Copyright (C) 2021 Meng Wang and Guizhen Lu. This is an open access article distributed under the Creative Commons Attribution License, which permits unrestricted use, distribution, and reproduction in any medium, provided the original work is properly cited.

\begin{abstract}
The contrast source inversion (CSI) is an effective method for solving microwave imaging problems which is widely utilized. The core of the CSI is to change the conventional inverse scattering problem into an optimization problem. The two items in the objective function describe the state error and data error, respectively. As it is all known, there is almost no complete performance comparison based on Fresnel data for the CSI and its related improved algorithms. In addition, the performance of the algorithm under different weights was not analyzed before and the convergence speed of original CSI is slow. Firstly, this paper compares the performance of traditional CSI and its improved algorithms from three aspects of qualitative imaging effect, convergence speed, and objective function value based on Fresnel data. Secondly, the influence of the state error and the data error under different weights on the convergence rate and the objective function value are studied. For the limitation of a slower convergence rate, the CSI with weights (W-CSI), the CSI with dynamic reduction factor (CSI-DRF), and its related algorithms, which can get better convergence rate compared with their relative original algorithms, are proposed. Eventually, the future research work is prospected.
\end{abstract}

\section{Introduction}

The technology of microwave imaging uses the obtained scattered field data to reconstruct the geometric shape, position, and material parameters of scatterers [1]. In recent years, it has been widely employed in biomedicine, earth remote sensing, nondestructive testing, and other fields [2]. Most microwave imaging algorithms are based on the Lippmann-Schwinger equation. Due to the highly nonlinear and ill-posed characteristic of this equation, the solution and calculation process of it are extremely challenging [3].

Based on the theoretical research of electromagnetic inverse scattering, a variety of microwave imaging algorithms have been proposed. Currently, the microwave imaging algorithms commonly utilized are mainly divided into three categories. The first type is stochastic optimization algorithm, such as evolution strategy [4], particle swarm optimization [5], and whale optimization algorithm [6]. This type of algorithms converts the microwave imaging problem into an optimization problem and obtains the result by continuous iterating. The second type of algorithms is based on the gradient optimization theory [7-9]. This type of algorithm has lower computational cost and smaller calculation error. The third type of algorithms is based on neural networks. With the continuous development of deep learning technology, the solution of microwave imaging is gradually combined with this emerging technology [10-12].

The contrast source inversion (CSI) is a classic microwave imaging algorithm that was proposed by Van den Berg and Kleinman in 1997 [13]. On the basis of the traditional data error equation, the state error equation was introduced. This item played a regularizing role, facilitating precise search. To a certain extent, a stable solution to the microwave imaging problem could be obtained. 
As it is all known, the Fresnel data is often applied to test the performance of microwave imaging algorithms [14]. There is almost no complete performance test of inversion algorithms based on the Fresnel data in the literatures related to the CSI and its correlative modified algorithms. In addition, there is almost no research for the different weights in the two terms of original objective function. In fact, although the data error and state error under different weights will not significantly affect the qualitative imaging results, it will affect the convergence rate and the value of the objective function obviously. Meanwhile, the original CSI has the drawback of a slower convergence speed $[15,16]$.

Firstly, this paper compares the performance of the contrast source inversion and its improved algorithms in terms of qualitative imaging results, objective function value, and convergence speed based on the Fresnel scattering data. Secondly, the influence of data error and state error under different weights on the convergence rate and the value of the objective function are discussed. Then, the weight and dynamic reduction factor (DRF) are introduced to improve the convergence value of original CSI and cross-correlated contrast source inversion (CC-CSI). The results prove that the proposed improved methods can obtain the better convergence rate.

The structure of paper is arranged as follows. In Section 2 , the basic model of the microwave imaging problem is described. In Section 3, the original CSI and its improved algorithms are described. In Section 4, the performance of different algorithms is compared based on Fresnel data. In Section 5, the influence of the state error and the data error under different weights is studied. The improved methods for the original algorithms are proposed.

\section{The Forward Model of Scattering Field}

The research object of the microwave imaging problem is the unknown scatterer located in the domain of interest (DOI). The data of the field outside the area can be obtained through the receiving antennas, and the information of the unknown object is calculated through inversion algorithms. The basic model of microwave imaging is shown in Figure 1.

Taking the two-dimensional microwave imaging problem as an example, the radiation characteristics of the incident antenna are usually known, so the distribution of incident field $E_{\text {inc }}$ in the DOI can be obtained by calculation. An unknown scatterer in a certain time-harmonic electromagnetic wave will excite a secondary electromagnetic field in the whole space due to electromagnetic induction and polarization, that is, the scattering field $E_{\text {sca }}$. The scattered field is superimposed on the incident field to obtain the total field $E_{\text {tot }}[17]$ :

$$
E_{\mathrm{tot}}=E_{\mathrm{inc}}+E_{\mathrm{sca}} \text {. }
$$

According to the definition of the incident field, the Helmholtz equation is obviously satisfied:

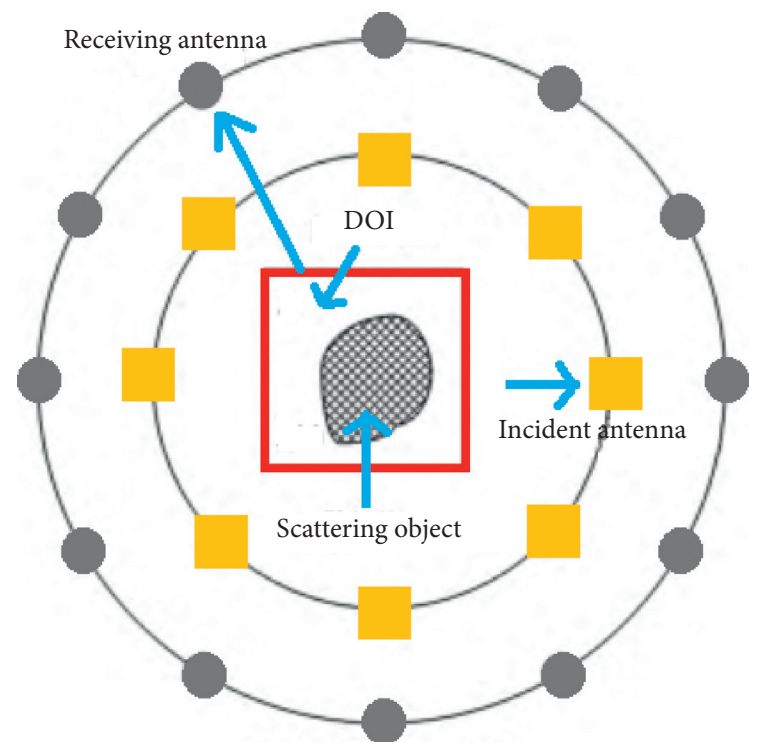

FIgURE 1: The basic model of microwave imaging.

$$
\nabla^{2} E_{\text {inc }}+k_{0}^{2} E_{\text {inc }}=0,
$$

where $k_{0}=\omega \sqrt{\varepsilon_{0} \mu_{0}}$.

According to Maxwell's equations, the total field satisfies the following relationship:

$$
\nabla^{2} E_{\text {tot }}+k_{0}^{2} E_{\text {tot }}=-i \omega \mu_{0} J+\nabla\left(\nabla \cdot E_{\text {tot }}\right) .
$$

Vector operation relations are used in the derivation process:

$$
\nabla \times(\nabla \times E)=\nabla(\nabla \cdot E)-\nabla^{2} E
$$

According to (1)-(4), the scattered field satisfies the following equation:

$$
\nabla^{2} E_{\mathrm{sca}}+k_{0}^{2} E_{\mathrm{sca}}=-i \omega \mu_{0}\left(\overline{\bar{I}}+\frac{\nabla \nabla}{k^{2}}\right) J .
$$

Then, the Dyadic Green function is employed. The solution of the scattering field is obtained as follows:

$$
E_{\mathrm{sca}}(r)=i \omega \mu_{0} \int_{D} G\left(r, r^{\prime}\right) J\left(r^{\prime}\right) \mathrm{d} r^{\prime}
$$

Eventually, the Lippmann-Schwinger equation describing the phenomenon of electromagnetic scattering is derived:

$$
E_{\text {tot }}(r)=E_{\text {inc }}(r)+i \omega \mu_{0} \int_{D} G\left(r, r^{\prime}\right) J\left(r^{\prime}\right) \mathrm{d} r^{\prime}
$$

It is difficult to obtain an analytical solution to the above integral equation, and generally, only the discretization method can be used to transform the integral equation into the linear equation to obtain approximate numerical solutions. Commonly used discretization methods are the method of moments (MoM), finite difference time domain 
(FDTD), finite difference frequency domain (FDFD), and so on [18].

\section{CSI and Its Improved Algorithms}

A novel construction method of the objective function was given by the CSI method [13]. In the contrast source inversion, data error and state error are defined as follows:

$$
\begin{aligned}
F_{\text {Data }} & =\frac{\sum_{j}\left\|E_{j}^{\text {sca }}-G^{S} \omega_{j}\right\|^{2}}{\sum_{j}\left\|E_{j}^{\text {sca }}\right\|^{2}}, \\
F_{\text {State }} & =\frac{\sum_{j}\left\|\chi E_{j}^{\text {inc }}-\omega_{j}+\chi G^{D} \omega_{j}\right\|^{2}}{\sum_{j}\left\|\chi E_{j}^{\text {inc }}\right\|^{2}} .
\end{aligned}
$$

The final objective function is the sum of two terms:

$$
\begin{gathered}
F_{\text {CSI }}=F_{\text {Data }}+F_{\text {State }}=\frac{\sum_{j}\left\|E_{j}^{\text {sca }}-G^{S} \omega_{j}\right\|^{2}}{\sum_{j}\left\|E_{j}^{\text {sca }}\right\|^{2}} \\
+\frac{\sum_{j}\left\|\chi E_{j}^{\text {inc }}-\omega_{j}+\chi G^{D} \omega_{j}\right\|^{2}}{\sum_{j}\left\|\chi E_{j}^{\text {inc }}\right\|^{2}} .
\end{gathered}
$$

The first term measures the error in the data equation and the second term measures the error in the object equation [19].

The performance of original CSI can be improved by combining with regularization methods [15]:

$$
F_{\mathrm{MR}-\mathrm{CSI}}=F_{\mathrm{CSI}} F_{\mathrm{MR}} \text {. }
$$

In (10), $F_{\mathrm{MR}}$ is the regularization factor, which is defined as follows:

$$
F_{\mathrm{MR}}=\frac{1}{S} \int_{D} \frac{|\nabla \chi|^{2}+\sigma_{n-1}^{2}}{\left|\nabla \chi_{n-1}\right|^{2}+\sigma_{n-1}^{2}} \mathrm{~d} r,
$$

where $S$ is the area of domain $D$ and $\sigma_{n-1}^{2}$ is the parameter that controls the effect of the regularization.

Cross-correlated CSI (CC-CSI) is a novel modified algorithm which was proposed in 2017. In the CC-CSI, an additional error term, which correlates the data and state errors, is added to the cost functional of original CSI.

This additional error term is defined as follows [16]:

$$
F_{\mathrm{CC}_{j}}=E_{\mathrm{sca}}-G_{s}\left(\chi E_{\mathrm{inc}}+\chi G_{D} \omega_{j}\right) .
$$

Therefore, the improved cost function for CC-CSI is defined as follows [20]:

$$
F_{\mathrm{CC}-\mathrm{CSI}}=F_{\mathrm{CSI}}+\frac{\sum_{j}\left\|F_{c c_{j}}\right\|_{S}^{2}}{\sum_{j}\left\|E_{\mathrm{sca}}\right\|_{S}^{2}} .
$$

\section{Experimental Results and Comparisons}

In this section, to measure the performance of the original CSI and its correlative improved algorithms MR-CSI and CC-CSI, the Fresnel data, which is chosen from [14], is applied. Qualitative imaging results, objective function value, and convergence speed are applied to measure the algorithms' performance. The Fresnel data comes from actual measurement in the microwave anechoic chamber. The type of scatterers and the field parameter settings are explained in detail in [14]. The data support for the research [14] can be found in the website link, which is from the literature.

In addition, the objective function values are obtained according to (9), (10), and (13), respectively.

4.1. "U-shaped" Groove. The "U-shaped" groove is a structure commonly utilized for the verification of imaging algorithms. Because of the obvious phenomenon of field coupling inside the groove, this structure is very suitable for measuring the performance of the imaging algorithms. The imaging results are shown in Figure 2.

Figures 2(a)-2(c) are the reconstruction results of contrast source for CC-CSI, MR-CSI, and CSI successively. The order and meaning of the figures below are arranged according to this rule.

It can be seen from the imaging results that the field coupling in the " $U$-shaped" groove is a difficulty for the current imaging methods. Although CC-CSI can reconstruct the shape of the " $U$-shaped" groove well, the imaging result is not very good in the coupling area in the groove. Due to the addition of the regular term, MR-CSI can constrain the objective function well. Therefore, the better imaging results for the coupling area in the groove can be obtained. The original CSI cannot obtain the shape characteristics of the " $U$-shaped" groove. The objective function values are shown in Figure 3:

It is obvious that although the original CSI can obtain the smallest objective function value and the fastest convergence rate among the three algorithms, it cannot obtain good imaging results. The existence of regularization in MR-CSI and CC-CSI can effectively improve imaging accuracy and reduce errors. MR-CSI can obtain the faster convergence speed and smaller objective function value.

4.2. Two Scatterers (Asymmetric). The imaging problem of two asymmetric regular scatterers is very classical. Due to its asymmetry, the scattering field distribution is not uniform, and there is also a coupling phenomenon between two scatterers, so it is particularly adapted for measuring the performance of imaging algorithms.

The imaging results are shown in Figure 4.

It can be seen from the imaging results that both CC-CSI and MR-CSI can reconstruct the position, the quantity, and the shape of the original scatterers. However, CSI can only reconstruct one scatterer. Some information of the scatterers has been missing through the reconstruction process of original CSI. The optimal function values are shown in Figure 5: 


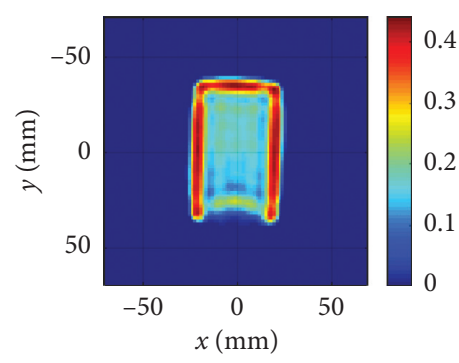

(a)

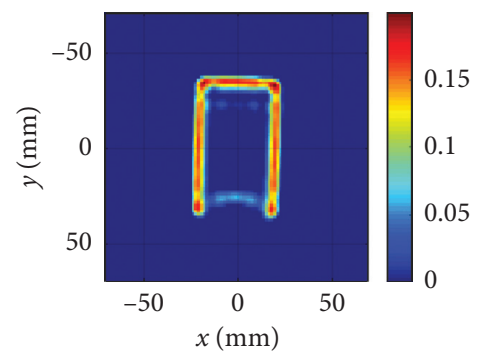

(b)

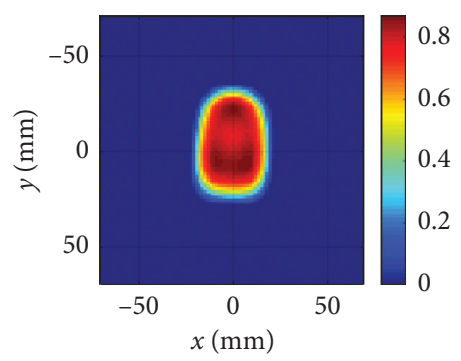

(c)

FIGURE 2: The imaging results of " $U$-shaped" groove.

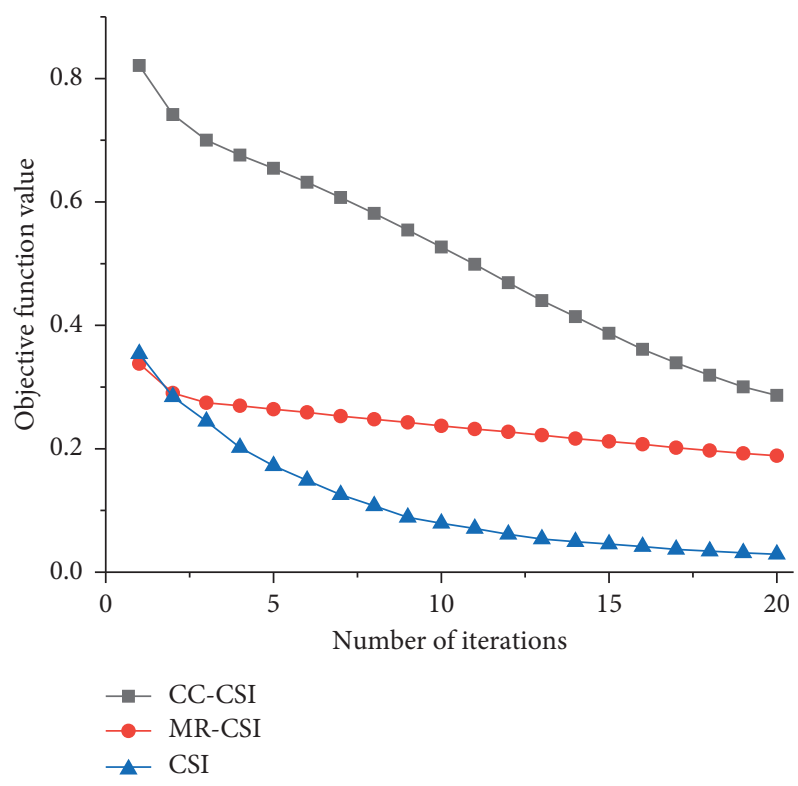

FIgURE 3: The objective function values of " $U$-shaped" groove.

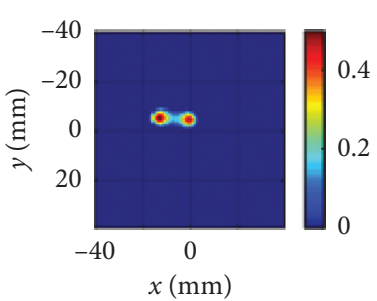

(a)

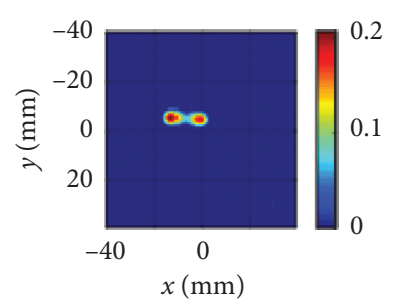

(b)

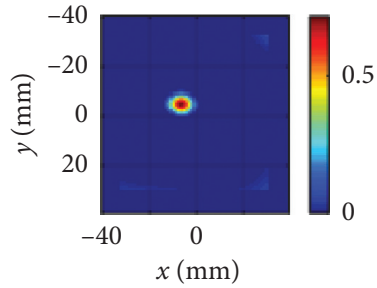

(c)

FIGURE 4: The imaging results of two scatterers (asymmetric).

It can be seen from the convergence curves that CSI has the fastest convergence speed and minimum objective function value. However, according to the comprehensive qualitative imaging results, this is a premature algorithm and cannot image scatter well. MR-CSI has achieved a faster convergence speed, and the performance is similar to CCCSI. MR-CSI can obtain the faster convergence speed and smaller objective function value.
4.3. Two Scatterers (Symmetry). Unlike B, the two scatterers employed in this section are symmetrical, which are shown in Figure 6. The field distribution of such symmetrical scatterers is more uniform than that of the asymmetrical scatterer in $B$.

It can be seen from the imaging results that both CC-CSI and MR-CSI can reconstruct the position, the amount, and the shape of the original scatterers. However, CSI only 


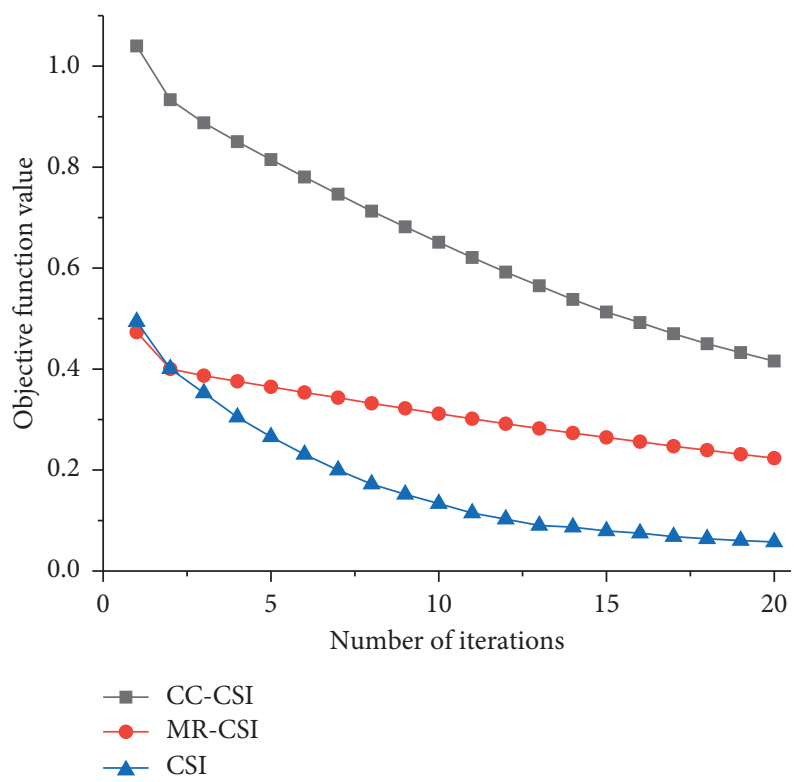

Figure 5: The objective function values of two scatterers (asymmetric).

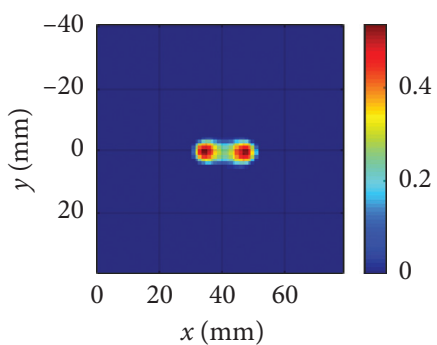

(a)

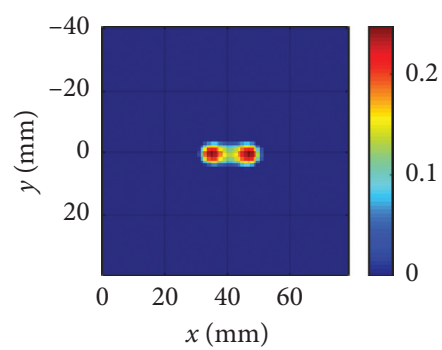

(b)

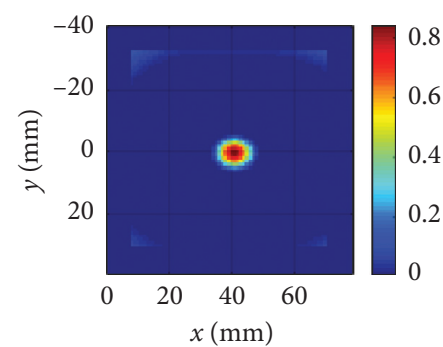

(c)

FIgURE 6: The imaging results of two scatterers (symmetry).

reconstructs one scatterer. The limitations of original CSI performance are almost identical to those in $B$. The objective function values are shown in Figure 7:

It can be seen from the convergence curves that CSI has the fastest convergence speed. The original CSI method does not have any additional regularization terms, so a smaller objective function value can be obtained. However, it does not play a good constraint on the solution of nonlinear equations, so the imaging performance is not good. MR-CSI can obtain the faster convergence speed and smaller objective function value.

4.4. Single Scatterer (TE Wave). The previous three sets of performance tests all use TM wave incidence. In this part, the TE wave incidence is applied. The results of imaging are shown in Figure 8.

TE waves have a magnetic field component but no electric field component in the propagation direction. Generally speaking, TE wave imaging is more difficult than the TM wave. From the imaging results, the three algorithms are not able to reconstruct the scatterers well. The objective function values are shown in Figure 9:
It can be seen from the convergence curve that CSI has the fastest convergence speed. MR-CSI can obtain the smallest objective function value. Compared with CC-CSI, MR-CSI can obtain a faster convergence speed and a smaller objective function value. The initial value of the iteration is very large.

4.5. Double Scatterers. The imaging problem of two regular scatterers, because of its relatively simple scattering theory, is widely used to compare the performance of related improvement algorithms for the imaging. The imaging results are shown in Figure 10.

It can be seen from the imaging results that all the algorithms can reconstruct the position and shape of the original scatterers. The position, quantity, and material parameters of the scatterers have been well reconstructed. The objective function values are shown in Figure 11.

It can be seen from the curve of the objective function value that CSI has the fastest convergence speed. CC-CSI can obtain the faster convergence speed, and MR-CSI can obtain the smaller objective function value under intercomparison. 


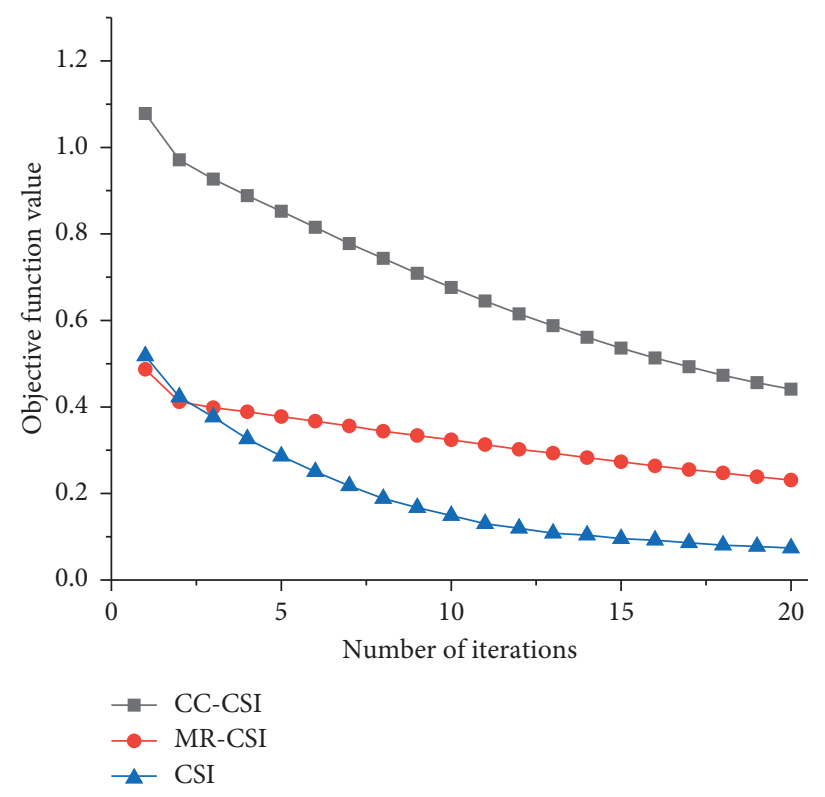

FIGURE 7: The objective function values of two scatterers (symmetric).

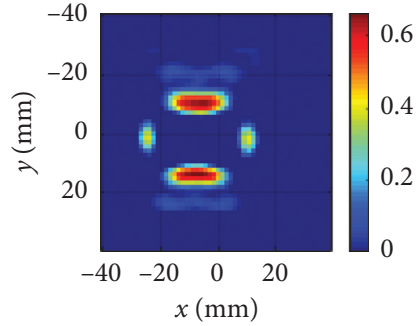

(a)

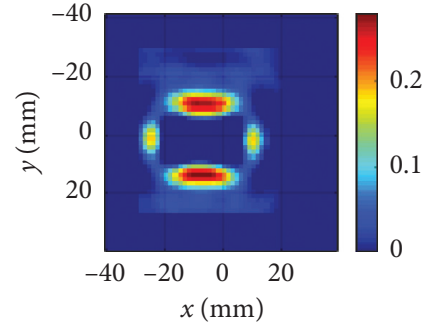

(b)

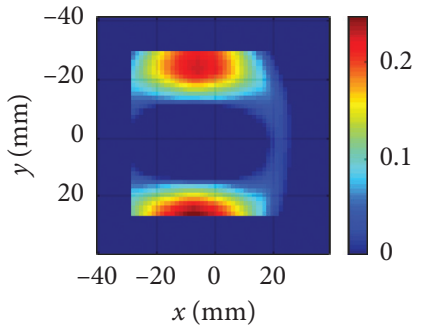

(c)

Figure 8: The imaging results of the single scatterer (TE wave).

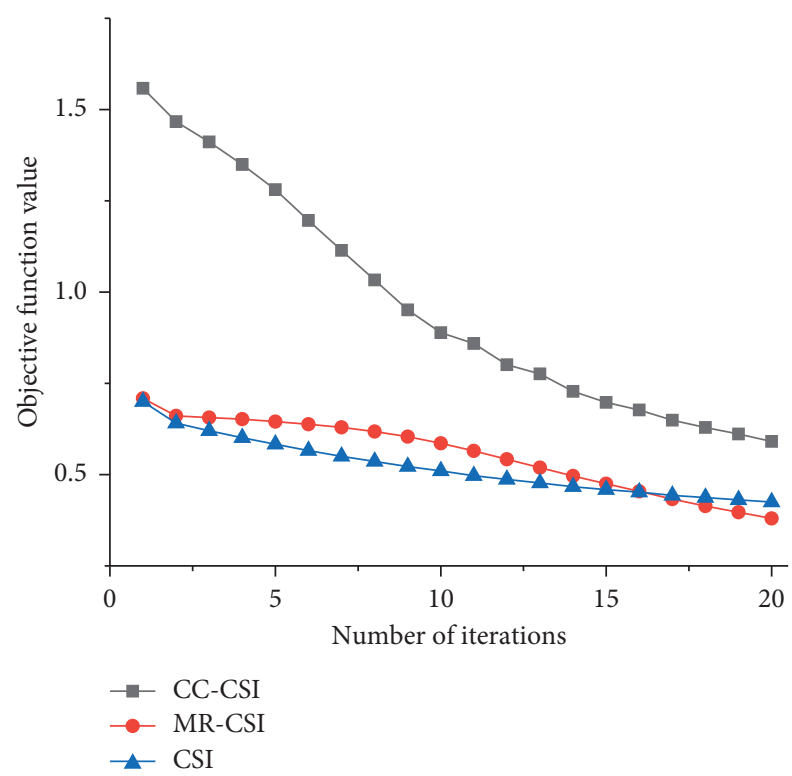

Figure 9: The objective function values of the scatterer (TE wave).
4.6. Single Scatterer. The microwave imaging problem of single and regular scatterer under multifrequency condition is relatively simple because the analytical solution of the scattered field can be accurately solved by the finite element method (FEM). The results of imaging are shown in Figure 12:

The results of imaging are shown in Figure 13.

It can be seen from the imaging results that all three algorithms can reconstruct the shape, position, and material parameters of the scatterer. And, it can be seen from the convergence curves that CSI has the fastest convergence speed. Because this kind of imaging problem is relatively simple, the original CSI does not contain any regular terms, so the fast convergence speed can be obtained.

In summary, according to the inversion results of Fresnel data above,

(1) The objective function of the original CSI is only composed of the state error and the data error. Therefore, in the process of iterative solution, a smaller convergence value can be obtained, compared to its improved algorithms. 


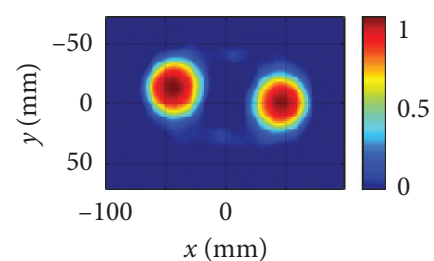

(a)

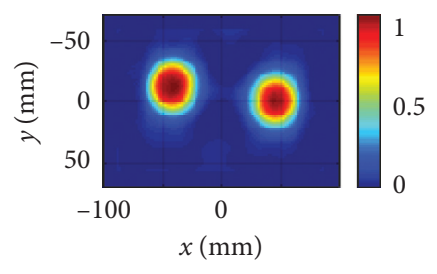

(b)

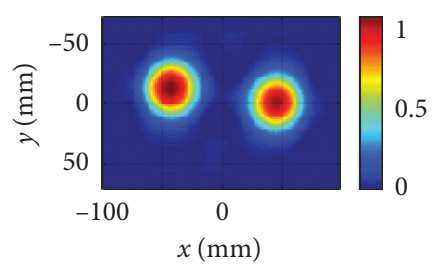

(c)

FIgURE 10: The imaging results of double scatterers.

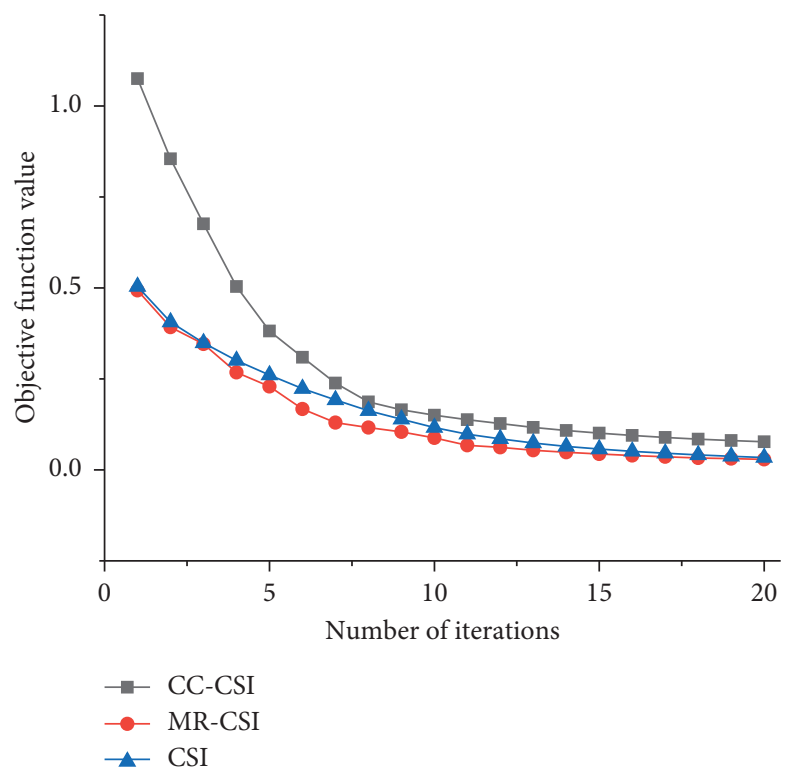

Figure 11: The objective function values of the double scatterers.

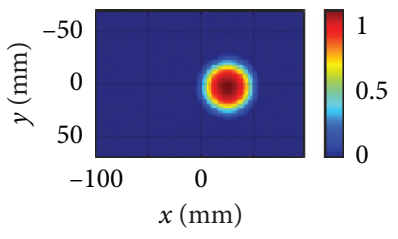

(a)

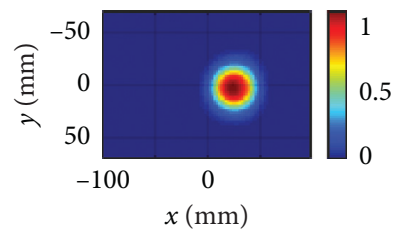

(b)

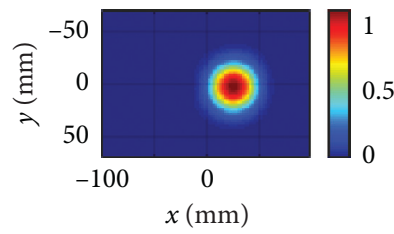

(c)

Figure 12: The imaging results of the single scatterer.

(2) Because there is no constraint of the regular terms, the original CSI cannot overcome the nonlinearity of the electric field integral equation (EFIE) well when solving some inverse scattering problems, and the solution result will be affected.

(3) According to the "no free lunch" theory, there is no single algorithm that can be applied to all situations. Therefore, in the microwave imaging problem, any algorithm has its specific advantages and disadvantages.

(4) The performance of original CSI and its improved algorithms cannot perform very well in solving the scatterers with field coupling and solving the problem of TE wave inversion.

(5) Although, in most cases, MR-CSI has better convergence speed and objective function value, the superiority and robustness of CC-CSI are still obvious considering the imaging effect, which has been proved in related literature.

\section{Influence of Different Weights for the Results}

In this section, firstly, the influence of different weights on the value of the objective function is analyzed, and then, two 


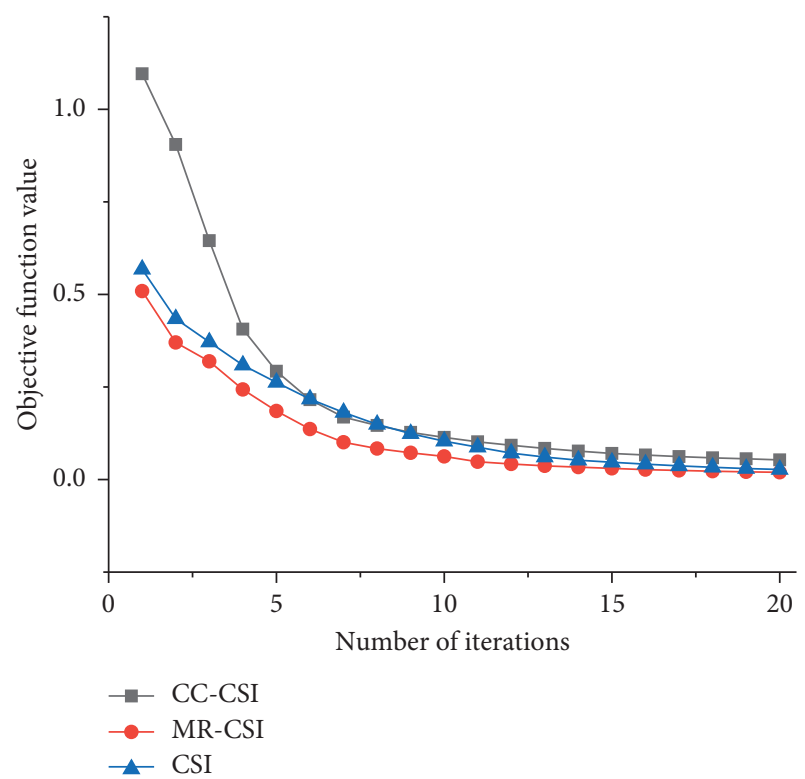

FIgURE 13: The objective function values of the single scatterer.

different improvement strategies are proposed for the slow convergence of original CSI.

5.1. The Analysis of Different Weights. In order to measure the influence of different weights on the imaging effect conveniently, a single and regular-shaped scatterer is utilized, which is shown in Figure 14. And, the details can also be found in [14]. (9).

The definition of objective function value is the same as

In order to discuss the influence of error terms with different weights on the convergence rate and objective function value, the objective function of the original CSI is improved as follows:

$$
F=\alpha \frac{\sum_{j}\left\|E_{j}^{\mathrm{sca}}-G^{S} \omega_{j}\right\|^{2}}{\sum_{j}\left\|E_{j}^{\mathrm{sca}}\right\|^{2}}+(1-\alpha) \frac{\sum_{j}\left\|\chi E_{j}^{\mathrm{inc}}-\omega_{j}+\chi G^{D} \omega_{j}\right\|^{2}}{\sum_{j}\left\|\chi E_{j}^{\mathrm{inc}}\right\|^{2}},
$$

where $\alpha$ and $1-\alpha$ are the coefficients of weight.

The different weight settings are shown in Table 1 . The weight setting includes a set of original objective functions without weights, six sets of different error weights, and two groups which are added with the square root operation based on the original objective function.

Figures 15(a)-15(i) correspond to group 1 to group 9 in the table, respectively. In order to facilitate the comparison of the influence of different weights on the convergence results clearly, all the curves are drawn together in Figure 16.

From the results shown in the figure, it can be seen that (h) and (i) are the convergence curves with the addition of the square root operation. The convergence curve is smooth, but the convergence value of the objective function is significantly increased.

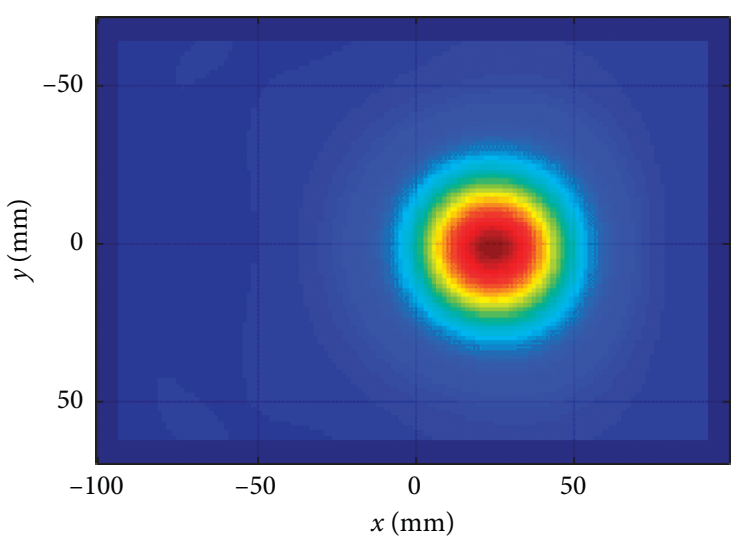

FIgURE 14: The single and regular-shaped scatterer.

TABle 1: The different settings of weight.

\begin{tabular}{lll}
\hline Group & $\alpha$ & $1-\alpha$ \\
\hline 1 & \multicolumn{2}{l}{ Original objective function } \\
2 & 0 & 1 \\
3 & 1 & 0 \\
4 & 0.4 & 0.6 \\
5 & 0.2 & 0.8 \\
6 & 0.6 & 0.4 \\
7 & 0.8 & 0.2 \\
8 & $\sqrt{F_{\text {Data }}+F_{\text {State }}}$ & \\
9 & $\sqrt{F_{\text {Data }}}+\sqrt{F_{\text {State }}}$ \\
\hline
\end{tabular}

Among the four convergence curves (d)-(g) with fixed weights, the initial value of curve (g) at the beginning of the iteration is larger, although the value of the objective function decreases rapidly. When the function value tends to be stable, a larger objective function value is still obtained. The initial value of curve (e) is small at the beginning of the iteration. Although the value of the objective function increases rapidly, when the value of the objective function tends to be stable, a smaller value of the objective function is still obtained.

For curve (e), the weight of its state error is greater than the data error weight; for curve $(g)$, the weight of its state error is smaller than the data error weight. Through the convergence results, it can be known that increasing the weight of the data error appropriately can effectively reduce the convergence value of the objective function.

In order to explore the influence of fixed weights on the imaging of scatterers with field coupling phenomenon, $U$ shaped groove is also used to detect performance. The convergence curves are shown in Figure 17. Obviously, the rule obtained from Figure 17 is the same with Figure 16.

The weights used above are all fixed weights. Inspired by the stochastic optimization algorithms, random weights are used, and the weights are random numbers between 0 and 2 . The scatterer is a $U$-shaped groove. The convergence curves are shown in Figure 18.

It can be seen from Figure 18 that, for CSI with random weights, no matter how the weights of state error and data error change, the value of the objective function fluctuates 


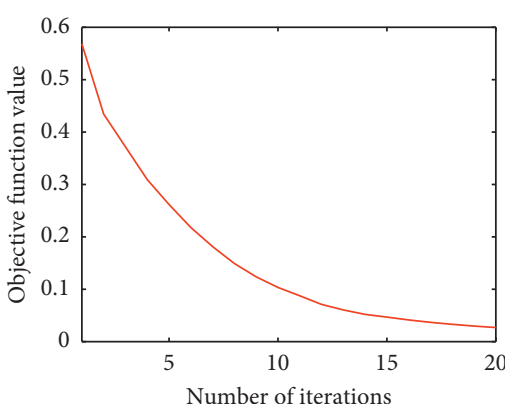

(a)

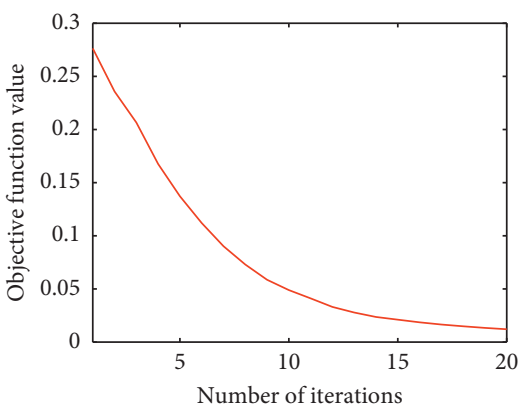

(d)

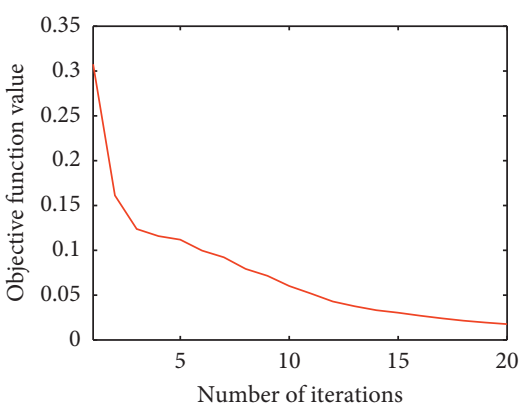

(g)

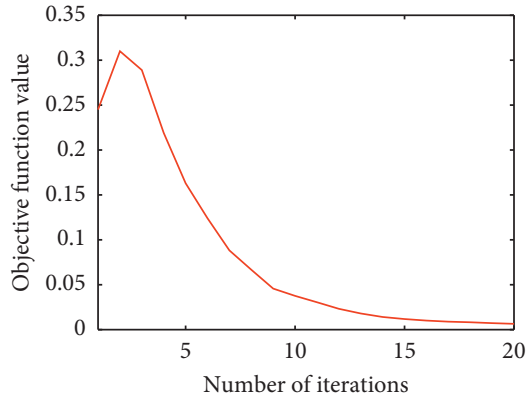

(b)

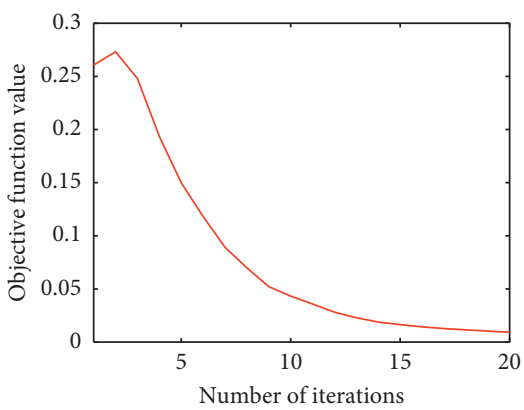

(e)

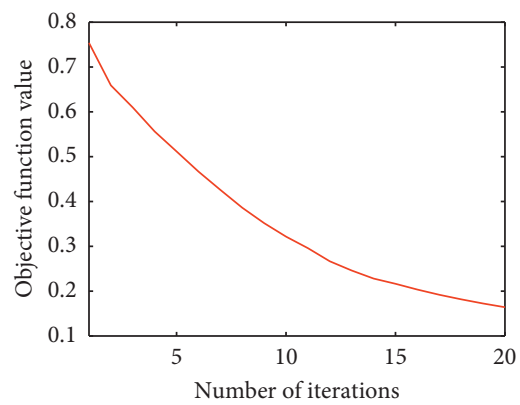

(h)

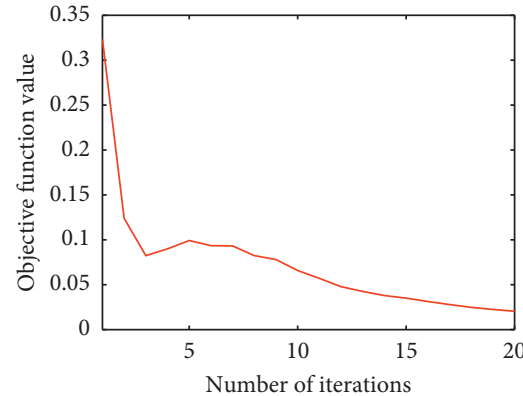

(c)

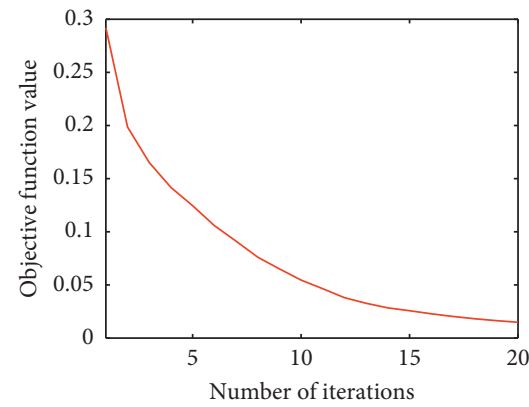

(f)

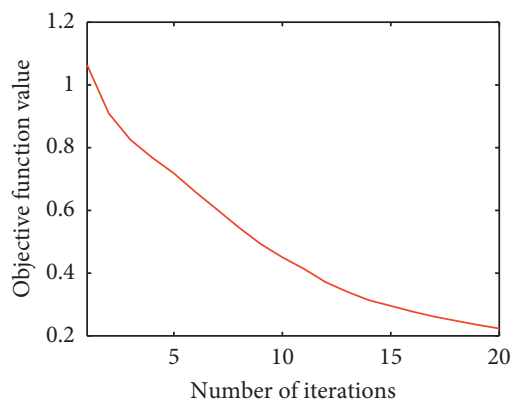

(i)

FIgURE 15: The convergence results with different weights.
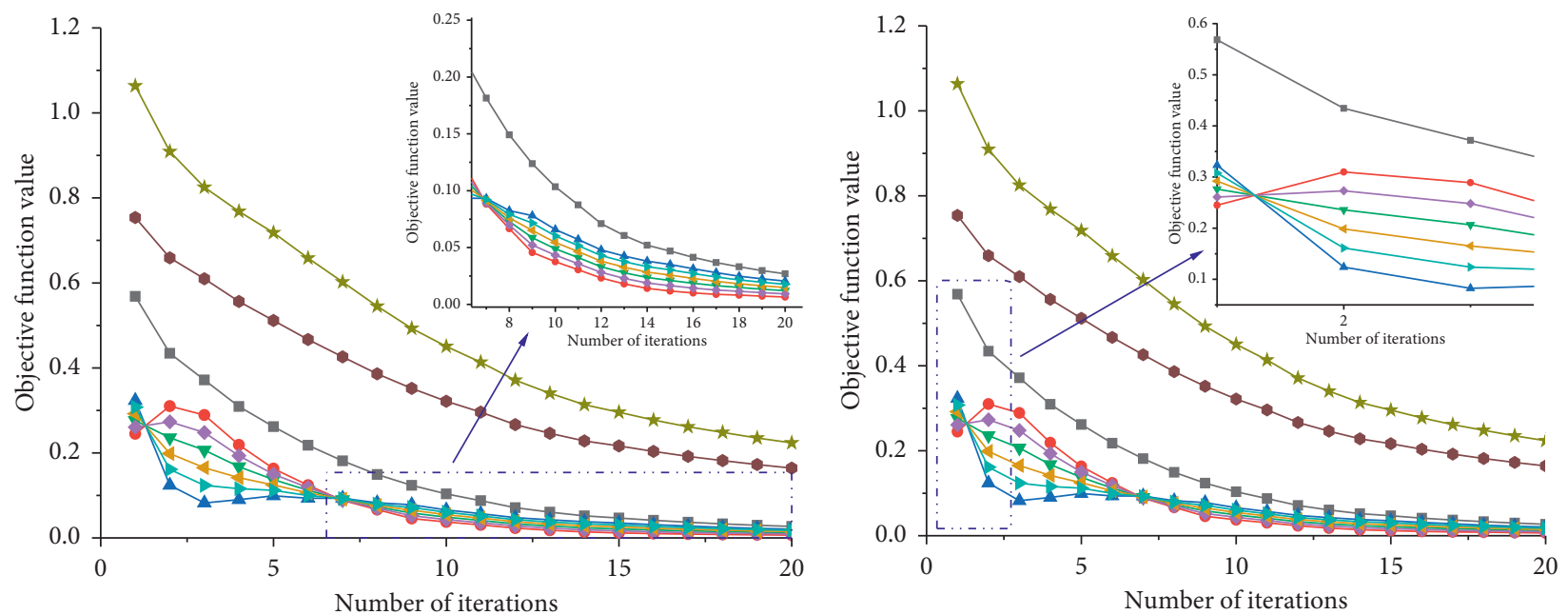

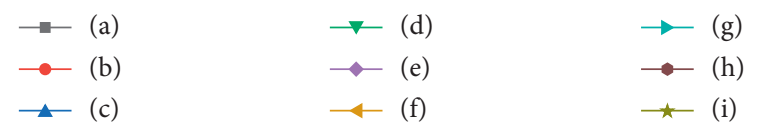

(a)

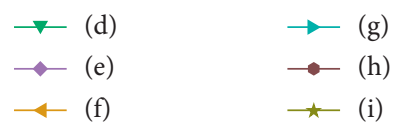

(b)

FIGURE 16: The convergence results of CSI with fixed weights for the regular scatterer. 


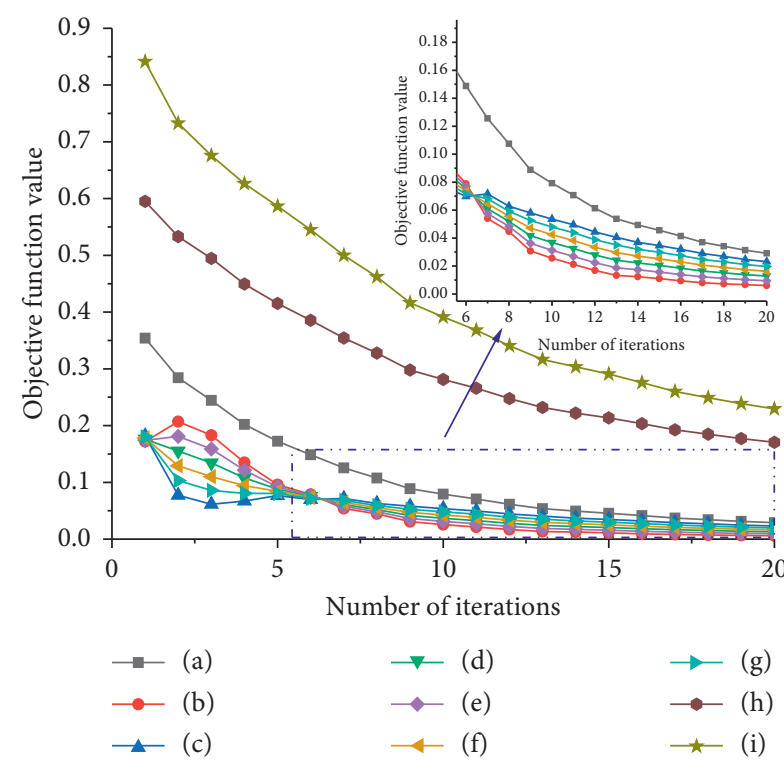

(a)

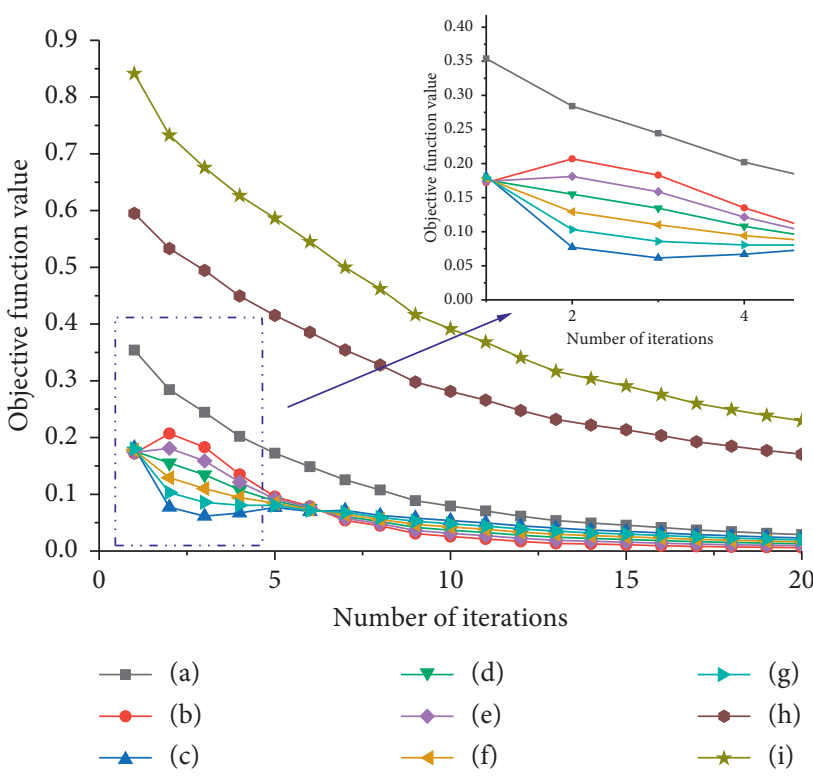

(b)

Figure 17: The convergence results of CSI with fixed weights for " $U$-shaped" groove.

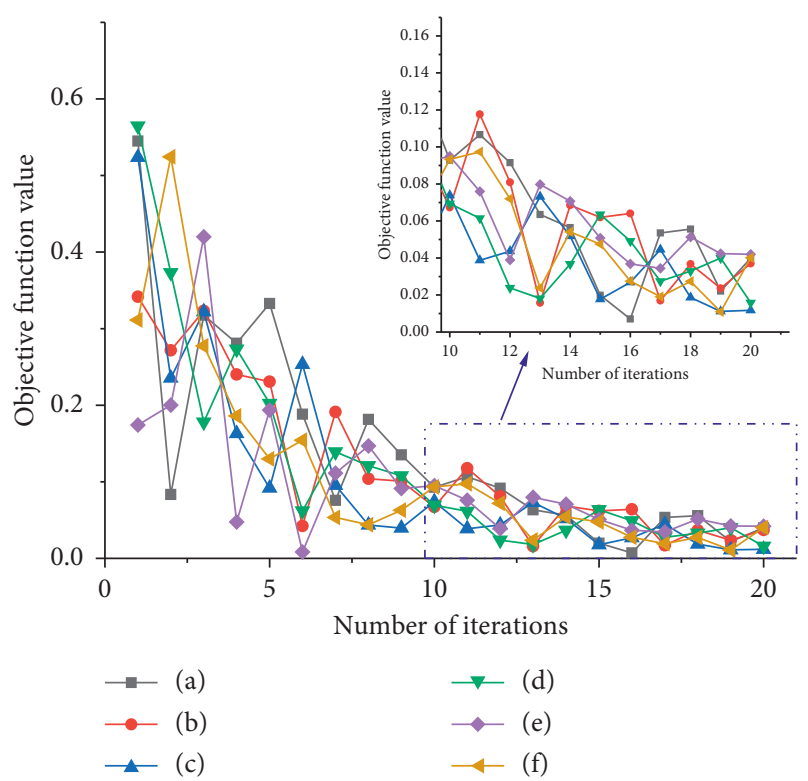

FIgURE 18: The convergence results of CSI with random weights.

greatly at the beginning of the iteration. Obviously, this is due to the uncertain weights in the iterative process. However, as the iteration continues, the change of the objective function gradually stabilizes and gradually tends to be 0 .

Cross-correlated contrast source inversion (CC-CSI) is a new type of improved CSI algorithms, which was proposed in 2017. First, fixed weights are utilized to explore the impact on the convergence speed of CC-CSI, and then, random weights are utilized, and the weight is a random number between $0-2$. The scatterers all employ $U$-shaped groove. The convergence results of CC-CSI with fixed weights for " $U$ shaped" groove are shown in Figure 19.

It can be seen from the figure that the convergence speed and objective function value of the traditional CC-CSI are the largest compared with the CC-CSI with weights. Among them, appropriately reducing the weight of the cross-correlation regular term is beneficial to improve the convergence speed and reduce objective function value.

The convergence results of CC-CSI with random weights for "U-shaped" groove are shown in Figure 20.

With random weights, the irregularity of the CC-CSI convergence curve matches the randomness of the weights exactly.

In summary, adding appropriate weights can effectively increase the convergence speed of the inversion algorithms and improve the value of the objective function.

5.2. The Algorithms with Weights. According to the rules summarized above and on the basis of traditional algorithms, two inversion algorithms with weights are proposed: contrast source inversion with weights (W-CSI) and cross-correlation contrast source inversion with weights (W-CC-CSI).

The objective function of W-CSI is defined as follows:

$$
F=\alpha \frac{\sum_{j}\left\|E_{j}^{\mathrm{sca}}-G^{S} \omega_{j}\right\|^{2}}{\sum_{j}\left\|E_{j}^{\mathrm{sca}}\right\|^{2}}+\beta \frac{\sum_{j}\left\|\chi E_{j}^{\mathrm{inc}}-\omega_{j}+\chi G^{D} \omega_{j}\right\|^{2}}{\sum_{j}\left\|\chi E_{j}^{\mathrm{inc}}\right\|^{2}},
$$

where $\alpha \in(0,0.5)$ and $\beta \in(0.5,1)$.

The objective function of W-CC-CSI is defined as follows: 


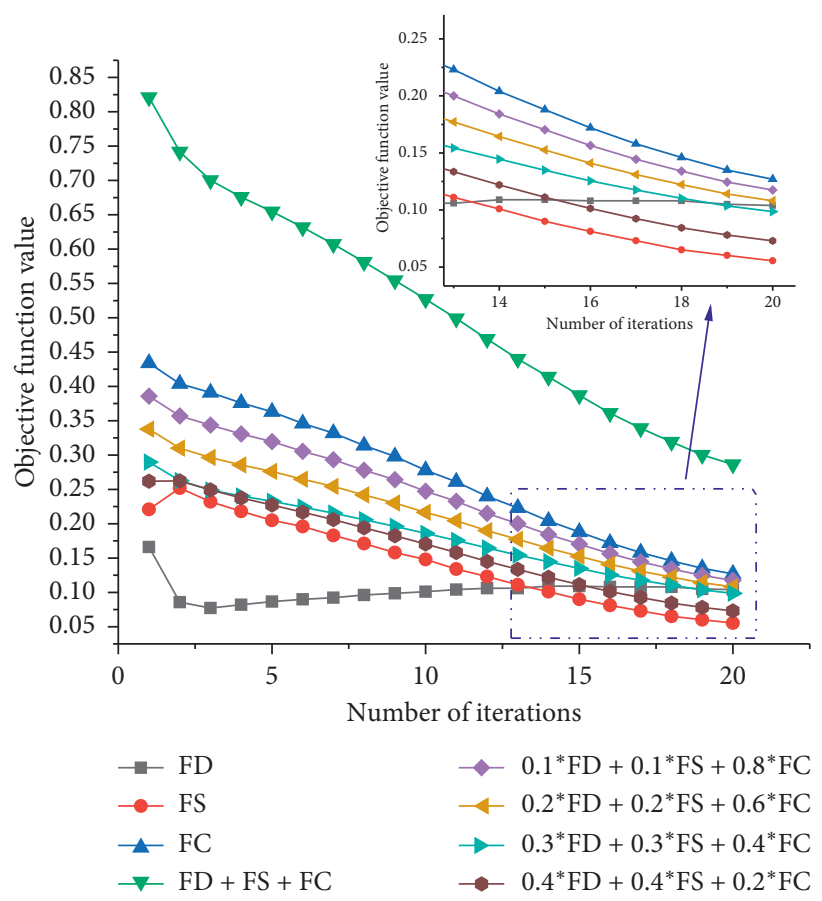

FIgURE 19: The convergence results of CC-CSI with fixed weights for " $U$-shaped” groove.

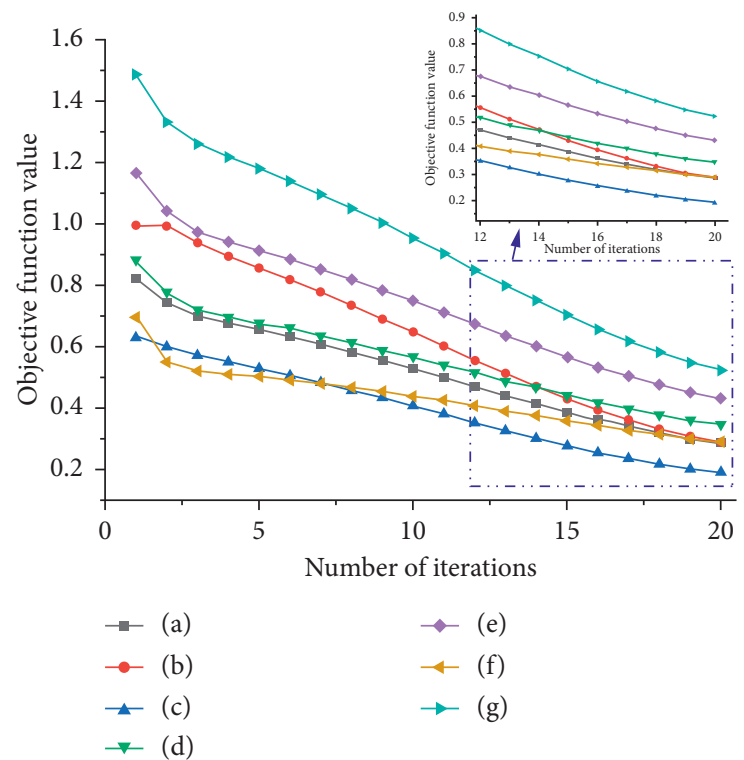

FIgURE 20: The convergence results of CC-CSI with random weights for " $U$-shaped" groove.

$$
\begin{aligned}
F= & \alpha \frac{\sum_{j}\left\|E_{j}^{\mathrm{sca}}-G^{S} \omega_{j}\right\|^{2}}{\sum_{j}\left\|E_{j}^{\mathrm{sca}}\right\|^{2}}+\beta \frac{\sum_{j}\left\|\chi E_{j}^{\mathrm{inc}}-\omega_{j}+\chi G^{D} \omega_{j}\right\|^{2}}{\sum_{j}\left\|\chi E_{j}^{\mathrm{inc}}\right\|^{2}} \\
& +\gamma \frac{\sum_{j}\left\|F_{c c_{j}}\right\|_{S}^{2}}{\sum_{j}\left\|E_{\mathrm{sca}}^{j}\right\|_{S}^{2}}
\end{aligned}
$$

where $\alpha \in(0,1), \beta \in(0,1), \gamma \in(0,1)$, and $\alpha, \beta>\gamma$.

First, take the $U$-shaped groove as an example to verify the performance of $\mathrm{W}$-CSI. The comparison of convergence curves is shown in Figure 21.

Then, take the $U$-shaped groove as an example to verify the performance of W-CC-CSI. The comparison of convergence curves is shown in Figure 22.

It can be seen from the convergence curves that the proposed W-CSI and W-CC-CSI can achieve faster 


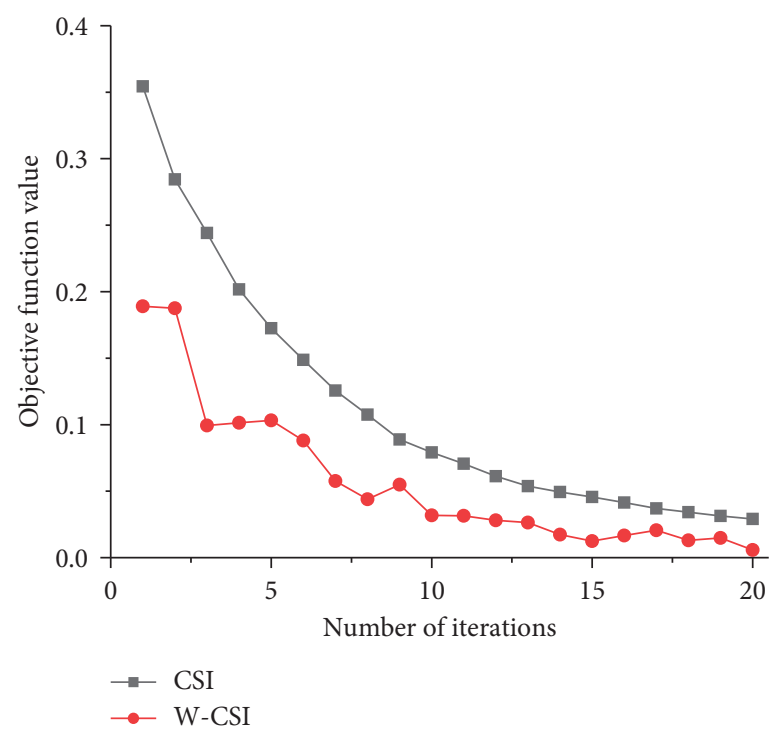

FIgURE 21: The comparison of convergence curves between CSI and W-CSI.

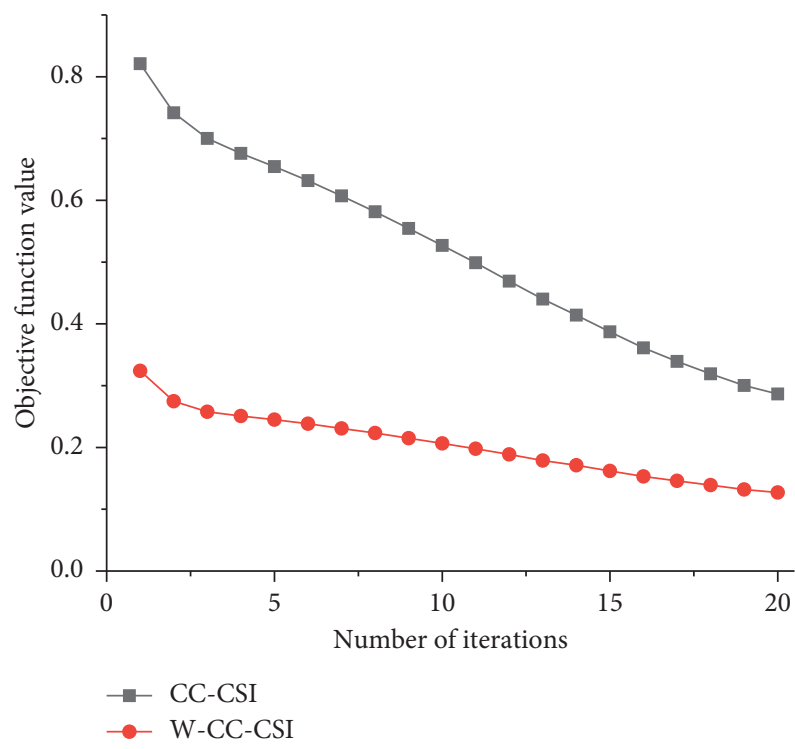

FIgure 22: The comparison of convergence curves between CC-CSI and W-CC-CSI.

convergence speed and smaller objective function value, compared with original CSI and CC-CSI.

5.3. The Algorithms with Dynamic Reduction Factor. In the field of stochastic optimization algorithms, convergence factor and dynamic reduction rate are methods commonly employed to improve convergence speed. For instance, Jia and $\mathrm{Lu}$ [21] proposed a new type of dynamic reduction rate, and it was applied to the antenna design. Good results could be obtained. CSI is a gradient-based inversion algorithm which can be combined with many different technologies to improve the performance. Therefore, the "dynamic reduction factor (DRF)" is also introduced in the traditional CSI inspired from the literature to improve the convergence speed, which is defined as follows:

$$
r=a *\left(1-\frac{t}{T}\right)^{2}
$$

where $a$ is a constant, $t$ is the current number of iterations, and $T$ is the maximum number of iterations. In this article, the value of $a$ is 1 .

In traditional CSI, the coefficients of the state error and the data error are both equal to 1 . The proposed dynamic reduction factor is a quadratic function that gradually equals to 0 as the number of iterations increases. As the iteration progresses, the step length of search can be reduced, and the oscillation phenomenon in the search can be effectively 


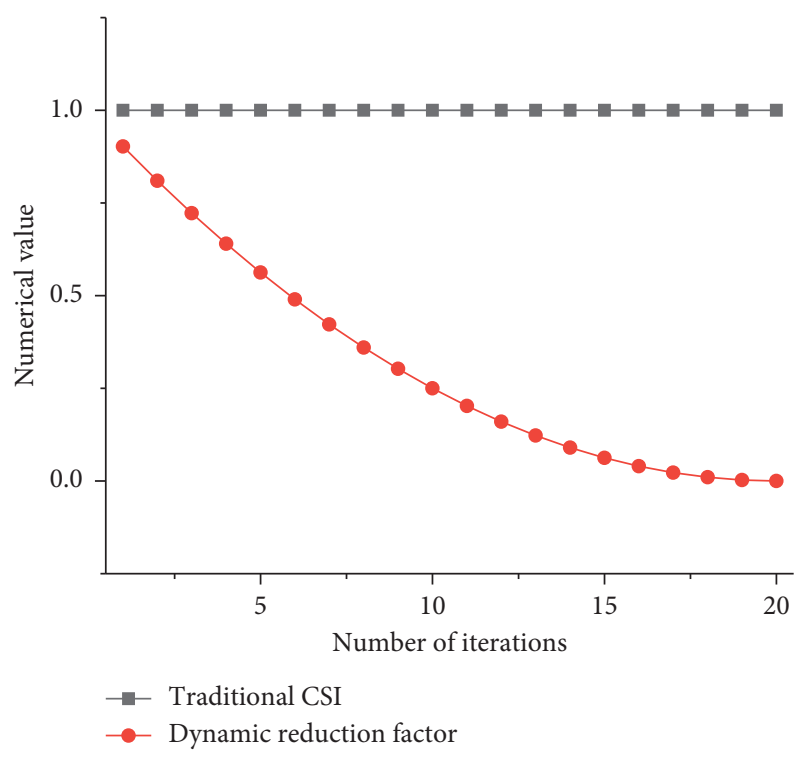

FIgURe 23: The comparison with the dynamic convergence factor.

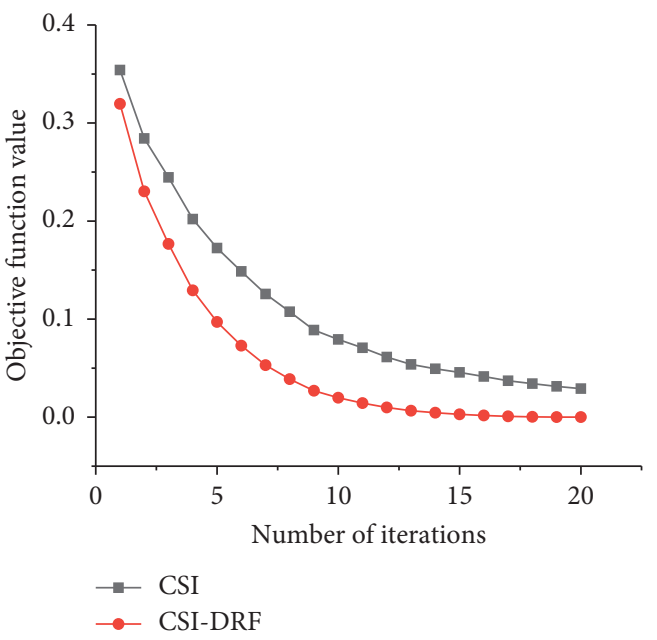

(a)

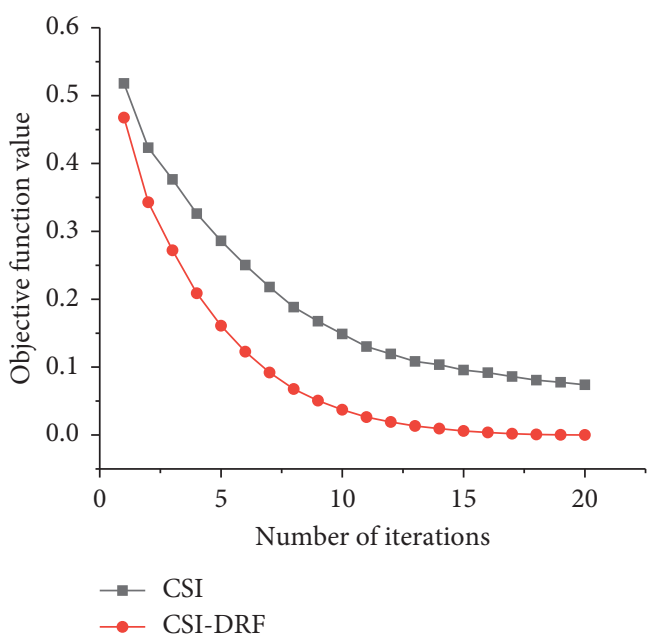

(c)

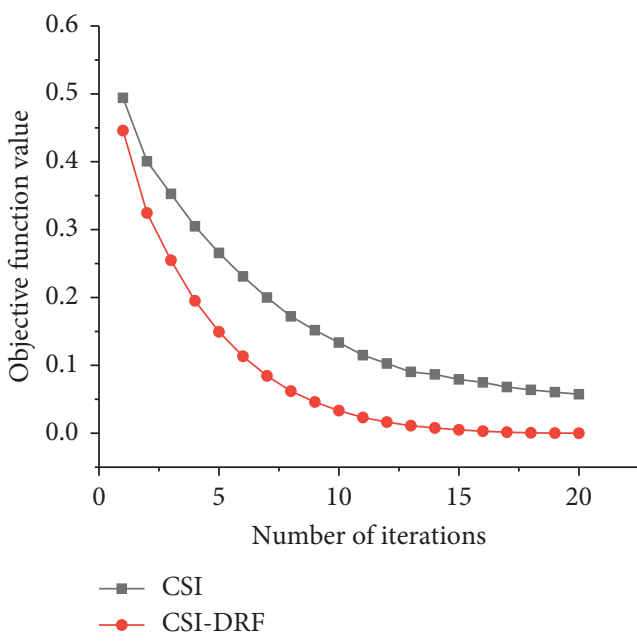

(b)

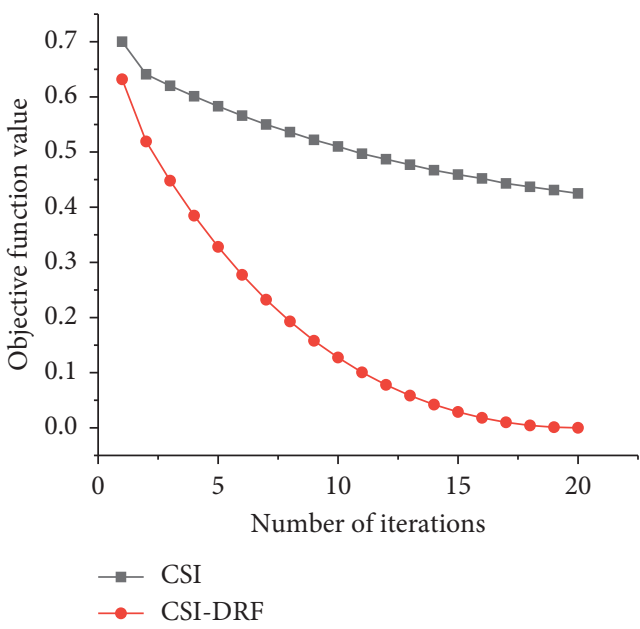

(d)

FIgURE 24: Continued. 


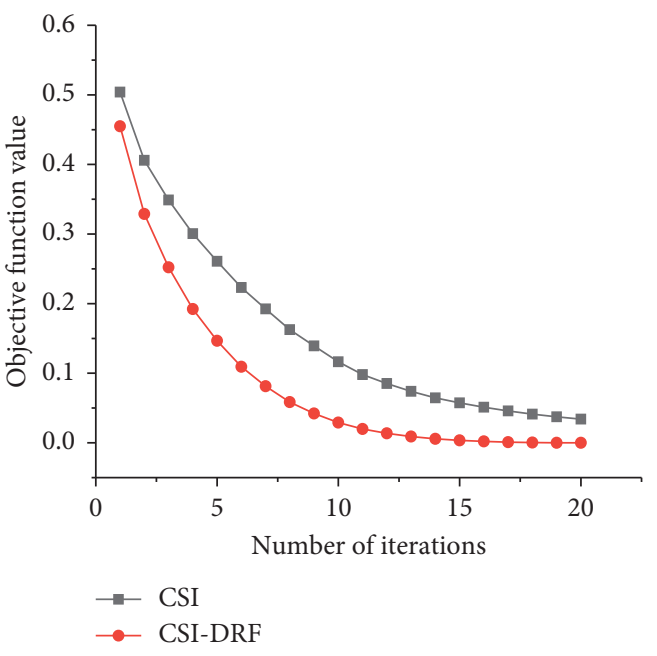

(e)

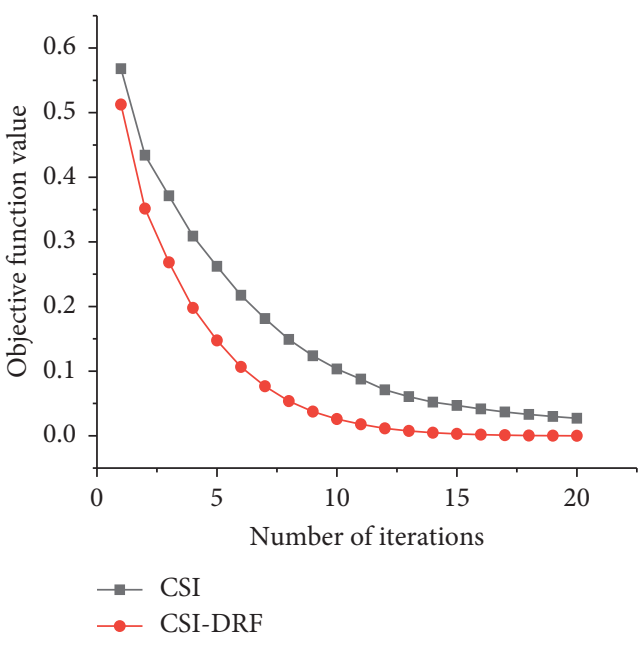

(f)

Figure 24: The comparison of convergence curves between CSI and CSI-DRF. (a) The comparison result of " $U$-shaped groove." (b) The comparison result of "two scatterers (asymmetric)." (c) The comparison result of "two scatterers (symmetry)." (d) The comparison result of "single scatterer (TE wave)." (e) The comparison result of "double scatterers." (f) The comparison result of "single scatterer."

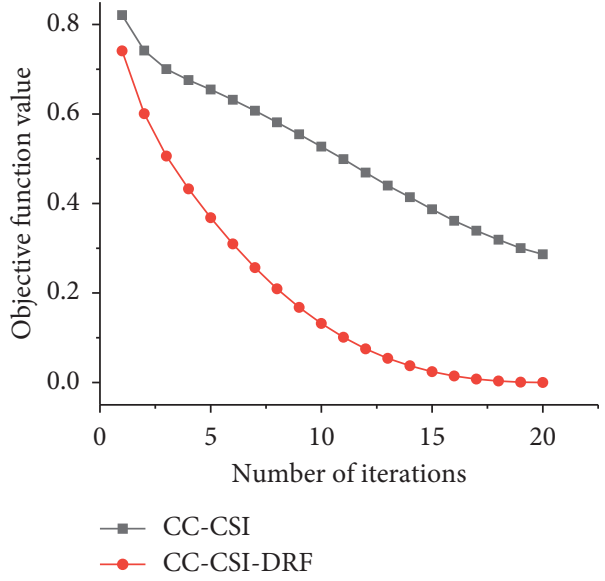

(a)

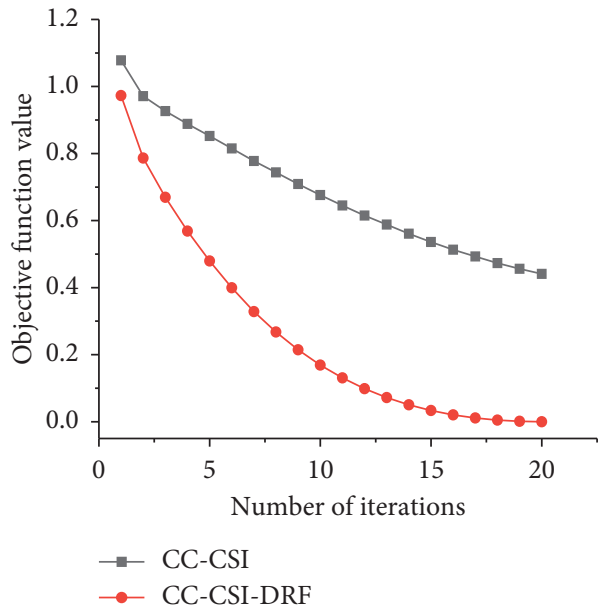

(c)

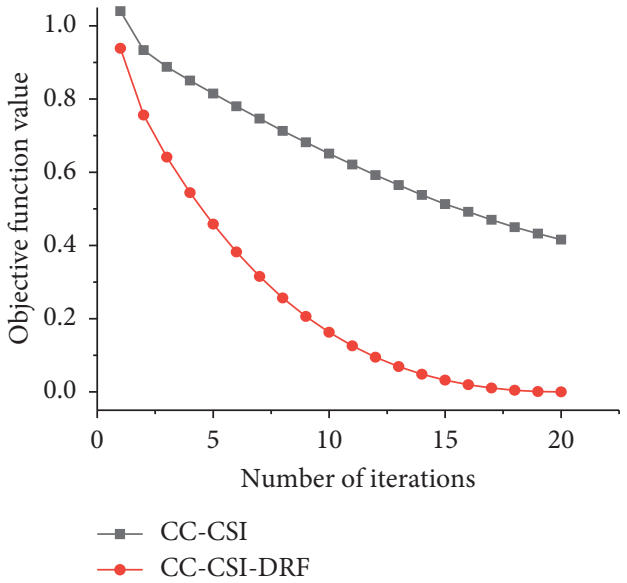

(b)

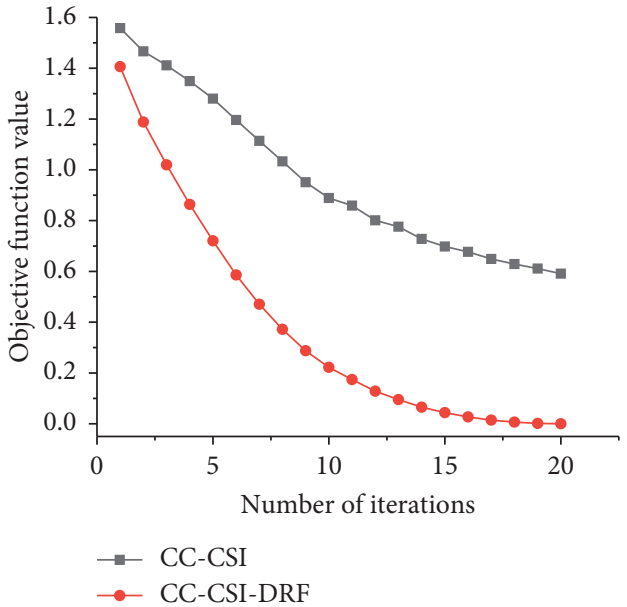

(d)

Figure 25: Continued. 


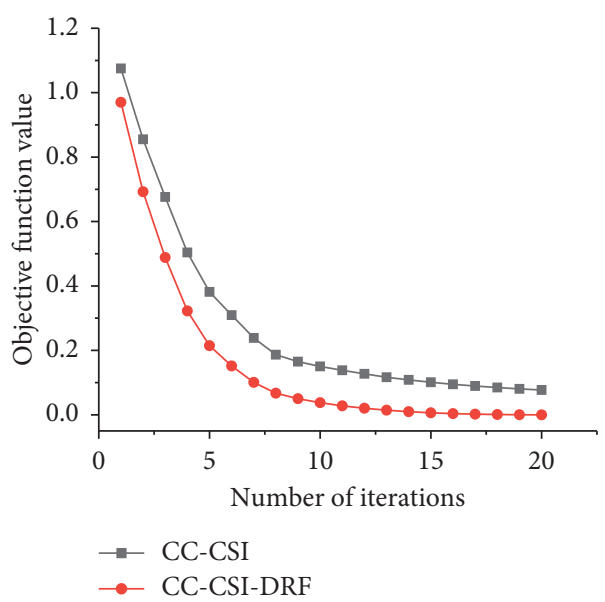

(e)

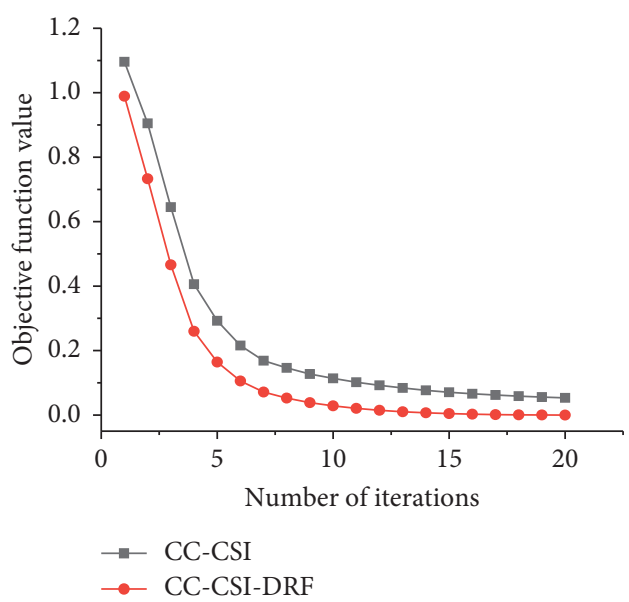

(f)

FIGURE 25: The comparison of convergence curves between CC-CSI and CC-CSI-DGF. (a). The comparison result of " $U$-shaped groove." (b). The comparison result of "two scatterers (asymmetric)." (c). The comparison result of "two scatterers (symmetry)." (d). The comparison result of "single scatterer (TE wave)." (e). The comparison result of "double scatterers." (f). The comparison result of "single scatterer."

reduced. To a certain extent, the introduction of DRF can change the distribution of isopleths, in order to better find the value of the objective function. It can be seen in Figure 23 that the DRF can achieve a faster convergence speed.
Then, the dynamic reduction factor is applied to the CSI, and the novel objective function is defined as follows:

$$
F=r *\left(\frac{\sum_{j}\left\|E_{j}^{\mathrm{sca}}-G^{S} \omega_{j}\right\|^{2}}{\sum_{j}\left\|E_{j}^{\mathrm{sca}}\right\|^{2}}+\frac{\sum_{j}\left\|\chi E_{j}^{\mathrm{inc}}-\omega_{j}+\chi G^{D} \omega_{j}\right\|^{2}}{\sum_{j}\left\|\chi E_{j}^{\mathrm{inc}}\right\|^{2}}\right) .
$$

In addition, the dynamic reduction factor is applied to the CC-CSI, and the novel objective function is defined as follows:

$$
F=r *\left(\frac{\sum_{j}\left\|E_{j}^{s c a}-G^{S} \omega_{j}\right\|^{2}}{\sum_{j}\left\|E_{j}^{s c a}\right\|^{2}}+\frac{\sum_{j}\left\|\chi E_{j}^{\text {inc }}-\omega_{j}+\chi G^{D} \omega_{j}\right\|^{2}}{\sum_{j}\left\|\chi E_{j}^{\text {inc }}\right\|^{2}}+\frac{\sum_{j}\left\|F_{c c_{j}}\right\|_{S}^{2}}{\sum_{j}\left\|E_{\text {sca }}^{j}\right\|_{S}^{2}}\right) .
$$

In order to measure the performances of the improved algorithms with the dynamic reduction factor, the same scatterers, as in Section 4, are applied for verification. The same detailed information of scatterers can be found in [14]. The results are shown in Figures 24 and 25.

It can be seen that the algorithms with the dynamic reduction factor CSI-DRF and CC-CSI-DRF can obtain the faster convergence speeds, and the convergence curves gradually tend to be zero, compared with the original algorithm CSI and CC-CSI.

\section{Conclusion}

In this paper, the performance of traditional CSI and its improved algorithms from three aspects of qualitative imaging effect, convergence speed, and objective function value based on Fresnel data are compared. It can be seen that the original CSI does not contain any regular terms, so it can obtain a faster convergence speed in the iterative process. However, in the face of seriously ill-posed inverse scattering problems, sometimes it cannot solve well. Although the introduction of the regular term effectively improves the imaging accuracy, the convergence speed is reduced. In addition, the inversion of TE wave is a problem that needs to be solved.

Secondly, the influence of the state error and the data error under different weights on objective function value and convergence rate are discussed. Fixed weights and random weights are used to verify the performance of the algorithms. The dynamic reduction factor (DRF) is introduced to improve the convergence rate. The CSI with weights (W-CSI), CC-CSI with weights (W-CC-CSI), CSI with dynamic 
reduction factor (CSI-DRF), and CC-CSI with dynamic reduction factor (CC-CSI-DRF) are proposed, which can get better convergence value and faster speed compared with their related original algorithms.

However, at the same time, the inverse scattering problem used in this article is relatively simple, and there are fewer types of scatterers, and no lossy materials are involved. There is one thing which should be noted. With the continuous development of artificial metamaterials, imaging algorithms for complex scatterers and complex environments have gradually become a research hotspot. In the future research work, the effective combination of emerging algorithms and related knowledge of complex electromagnetic scattering will be a new research goal.

In addition, the stochastic optimization algorithm has been well applied in engineering problems because of its good performance, such as butterfly optimization (MBO), earthworm optimization algorithm (EWA), and elephant herding optimization (EHO). In the future research, combining microwave imaging with these new optimization algorithms is also a research direction.

\section{Data Availability}

Some or all data, models, or code generated or used during the study are available from the corresponding author upon request.

\section{Conflicts of Interest}

The authors declare that they have no conflicts of interest.

\section{References}

[1] C. Xudong, Computational Methods for Electromagnetic Inverse Scattering, John Wiley \& Sons Singapore Pte. Ltd, Singapore, 2018.

[2] J. A. Devaney, Mathematical Foundations of Imaging, Tomography and Wavefield Inversion, Cambridge University Press, Cambridge, UK, 2012.

[3] M. Pastorino, "Stochastic optimization methods applied to microwave imaging: a review," IEEE Transactions on Antennas and Propagation, vol. 55, no. 3, pp. 538-548, 2007.

[4] L. Yang, S. Yang, S. L. Ho, and W. N. Fu, "An improved evolution strategy and its application to inverse scattering in microwave imaging," IEEE Transactions on Magnetics, vol. 51, no. 3, pp. 1-4, 2015.

[5] B. Mhamdi, "Microwave imaging based on two hybrid particle swarm optimization approaches," International Journal of Microwave and Wireless Technologies, vol. 11, no. 3, pp. 268-275, 2019.

[6] K.-C. Lee and P.-T. Lu, "Application of whale optimization algorithm to inverse scattering of an imperfect conductor with corners," International Journal of Antennas and Propagation, vol. 2020, Article ID 8205797, 9 pages, 2020.

[7] K. Xu, Yu Zhong, R. Song, X. Chen, and L. Ran, "Multiplicative-regularized FFT twofold subspace-based optimization method for inverse scattering problems," IEEE Transactions on Geoscience and Remote Sensing, vol. 53, no. 2, pp. 841-850, 2015.
[8] C. Xudong, "Subspace-based optimization method for solving inverse-scattering problems," IEEE Transactions on Geoscience \& Remote Sensing, vol. 48, no. 1, pp. 42-49, 2009.

[9] T. Yin, Z. Wei, and X. Chen, "Wavelet transform subspacebased optimization method for inverse scattering," IEEE Journal on Multiscale and Multiphysics Computational Techniques, vol. 3, pp. 176-184, 2018.

[10] M. T. Mccann, K. H. Jin, and M. Unser, "Convolutional neural networks for inverse problems in imaging: a review," IEEE Signal Processing Magazine, vol. 34, no. 6, pp. 85-95, 2017.

[11] Z. Wei and X. Chen, "Physics-inspired convolutional neural network for solving full-wave inverse scattering problems," IEEE Transactions on Antennas and Propagation, vol. 67, no. 9, pp. 6138-6148, 2019.

[12] Z. Wei and X. Chen, "Deep-learning schemes for full-wave nonlinear inverse scattering problems," IEEE Transactions on Geoscience and Remote Sensing, vol. 57, pp. 1849-1860, 2019.

[13] P. M. Van den Berg and R. E. Kleinman, "A contrast source inversion method," Inverse Problems, vol. 13, no. 6, pp. 1607-1620, 1997.

[14] K. Belkebir and M. Saillard, "Special section: testing inversion algorithms against experimental data," Inverse Problems, vol. 17 , no. 6, pp. 1565-1571, 2001.

[15] P. M. Van den Berg, A. Abubakar, and J. T. Fokkema, "Multiplicative regularization for contrast profile inversion," Radio Science, vol. 38, no. 2, p. 8022, 2003.

[16] S. Sun, B. J. Kooij, T. Jin, and A. G. Yarovoy, "Cross-correlated contrast source inversion," IEEE Transactions on Antennas and Propagation, vol. 65, no. 5, pp. 2592-2603, 2017.

[17] D. M. Pozar, Microwave Engineering, Publishing House of Electronics Industry, Beijing, China, 2004.

[18] F. Cakoni and D. Colton, Qualitative Methods in Inverse Scattering Theory, Springer Vienna, Berlin, Germany, 2006.

[19] P. M. Van den Berg and A. Abubakar, "Contrast source inversion method: state of art," Progress in Electromagnetics Research, vol. 34, pp. 189-218, 2001.

[20] S. Sun, B.-J. Kooij, and A. G. Yarovoy, "Inversion of multifrequency data with the cross-correlated contrast source inversion method," Radio Science, vol. 53, no. 6, pp. 710-723, 2018.

[21] X. Jia and G. Lu, "An improved random taguchi's method based on swarm intelligence and dynamic reduced rate for electromagnetic optimization," IEEE Antennas and Wireless Propagation Letters, vol. 18, no. 9, pp. 1878-1881, 2019. 